\title{
Emergence of atom-light-mirror entanglement inside an optical cavity
}

\author{
C. Genes, D. Vitali and P. Tombesi \\ Dipartimento di Fisica, Università di Camerino, I-62032 Camerino (MC), Italy
}

\begin{abstract}
We propose a scheme for the realization of a hybrid, strongly quantum-correlated system formed of an atomic ensemble surrounded by a high-finesse optical cavity with a vibrating mirror. We show that the steady state of the system shows tripartite and bipartite continuous variable entanglement in experimentally accessible parameter regimes, which is robust against temperature.
\end{abstract}

PACS numbers: 03.67.Mn, 85.85.+j,42.50.Wk,42.50.Lc

Recently there has been an increasing convergence between condensed matter physics and quantum optics, which has manifested in different ways. On one hand, systems of cold trapped atoms [1], ions [2] and electrons [3] may realize quantum simulators able to reproduce and study condensed matter concepts such as Fermi surfaces and Heisenberg models in a controllable and tunable way. On the other hand, circuit cavity QED [4] provides an example where nano- and microstructured condensed matter systems are specifically designed in order to reproduce the phenomena and control of quantum coherence typical of quantum optics system. Alternatively, one can design schemes in which one has a direct, strong coupling between an atomic degree of freedom and a condensed matter system. Examples of this latter kind are ion-nanomechanical oscillator [5], or ion-Cooper-pair box [6] systems, or a BoseEinstein condensate coupled to a cantilever via a magnetic tip [7]. A further important example is provided by cavity optomechanical systems for which strong coupling between an optical cavity mode and a vibrational mode by radiation pressure has been already demonstrated [8, 9, 10, 11, 12, 13, 14, 15, 16, 17, 18], and for which schemes able to show quantum entanglement [19, 20, 21, 22] and even quantum teleportation [23] have been already proposed. In these systems, the radiation pressure interaction can be made considerably large so that genuine quantum effects can be realized when microcavities and extremely light acoustic resonators [10, 11, 12, 13, 14, 15, 18] are used.

In this letter, we propose a hybrid system formed by an atomic ensemble placed within an optical Fabry-Perot cavity, in which a micromechanical resonator represents one of the mirrors [see Fig. 1(a)]. The atoms are indirectly coupled to the mechanical oscillator via the common interaction with the intracavity field. As a first step towards quantum state engineering of mechanical oscillators and quantum state transfer between atoms and mirrors, we show that using state-of-the-art technology it is possible to generate stationary and robust continuous variable $(\mathrm{CV})$ tripartite entanglement in the fieldatoms-mirror system. To this purpose, we consider $N_{a}$ two-level atoms placed in an optical cavity under weakcoupling conditions and far from the cavity main res- onance $\omega_{c}$. CV tripartite entanglement can be generated by choosing as working point for the optical cavity with vibrating mirror, the parameter regime corresponding to the ground state cooling of the mechanical resonator [24, 25, 26, 27]. In fact, preferential scattering of cavity light into a higher frequency motional sideband of the driving laser is responsible for cooling of the mechanical system. It has been shown in 22] that in this cooling regime, field-mirror entanglement can be generated, which can be explained in terms of sideband scattering because such an entanglement is mostly carried by the Stokes sideband. All these facts are at the basis of the robust tripartite atom-resonator-field entanglement reported here. In fact, if the laser anti-Stokes sideband is resonant with the cavity, the mechanical resonator is cooled by photon leakage and if then the atomic frequency matches the red (Stokes) sideband frequency, a resonant atoms-mirror coupling mediated by the cavity field is established. We shall see that in such a regime robust CV tripartite and bipartite entanglement is gen-

(a)

(b)

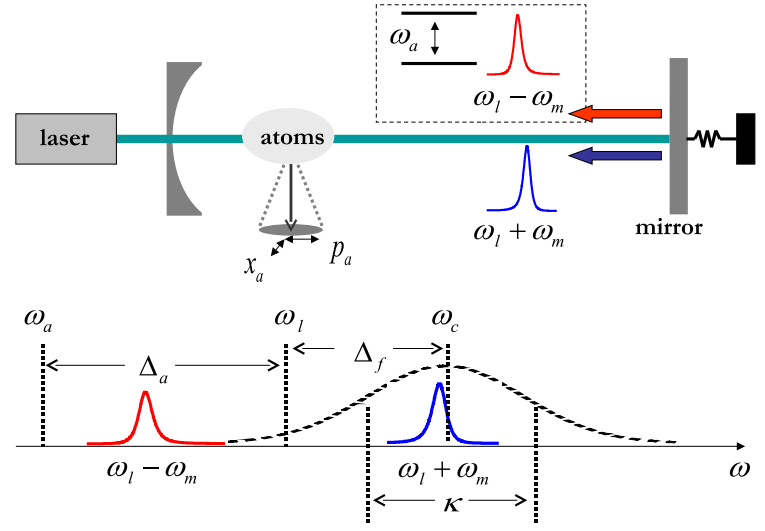

FIG. 1: (Color online) (a) The cavity is driven by a laser at frequency $\omega_{l}$ and the moving mirror at frequency $\omega_{m}$ scatters photons on the two sidebands at frequency $\omega_{l} \pm \omega_{m}$. (b) If the cavity with frequency $\omega_{c}$ and bandwidth $\kappa$, is put into resonance with the Antistokes sideband (blue), outgoing cavity photons cool the mirror vibrational mode. If the atoms are off-resonance with the cavity but resonantly coupled to the red sideband, an entangled tripartite atom-field-mirror system emerges. 
Description of the system. We consider an optical cavity with a fixed input mirror and a second oscillating mirror, which is driven by a laser at frequency $\omega_{l}$. An ensemble of two-level atoms is placed inside the cavity and it is off-resonantly coupled by a collective Tavis-Cummings type interaction to the optical field [28]. Mirror vibrational motion can be modeled by a harmonic oscillator of frequency $\omega_{m}$ and decay rate $\gamma_{m}$. In the absence of dissipation and fluctuations the total Hamiltonian of the system is given by the sum of a free evolution term

$$
H_{0}=\hbar \omega_{c} a^{\dagger} a+\frac{\hbar \omega_{a}}{2} S_{z}+\frac{\hbar \omega_{m}}{2}\left(q^{2}+p^{2}\right),
$$

and the interaction term

$$
\begin{aligned}
H_{I}= & \hbar g\left(S_{+} a+S_{-} a^{\dagger}\right)-\hbar G_{0} a^{\dagger} a q \\
& +i \hbar E_{l}\left(a^{\dagger} e^{-i \omega_{l} t}-a e^{i \omega_{l} t}\right) .
\end{aligned}
$$

The laser drives significantly only a single cavity mode with frequency $\omega_{c}$, bandwidth $\kappa$ and annihilation operator $a$ (with $\left[a, a^{\dagger}\right]=1$ ). The atomic ensemble is comprised of $N_{a}$ two-level atoms with natural frequency $\omega_{a}$ each described by the $1 / 2$ spin algebra of Pauli matrices $\sigma_{+}, \sigma_{-}$and $\sigma_{z}$. Collective spin operators are defined as $S_{+,-, z}=\sum_{\{i\}} \sigma_{+,-, z}^{(i)}$ for $i=1, N_{a}$ and satisfy the commutation relations $\left[S_{+}, S_{-}\right]=S_{z}$ and $\left[S_{z}, S_{ \pm}\right]= \pm 2 S_{ \pm}$. The mechanical mode dimensionless position and momentum operators $q$ and $p$ satisfy $[q, p]=i$. The atom-cavity coupling constant is given by $g=\mu \sqrt{\omega_{c} / 2 \hbar \epsilon_{0} V}$ where $V$ is the cavity mode volume and $\mu$ is the dipole moment of the atomic transition. The radiation pressure coupling constant is instead given by $G_{0}=\left(\omega_{c} / L\right) \sqrt{\hbar / m \omega_{m}}$, where $m$ is the effective mass of the mechanical mode, and $L$ is the length of the cavity. The last term describes the driving of the cavity by the laser with amplitude $E_{l}$, which is related to the input power $P_{l}$ and the cavity decay rate $\kappa$ by $\left|E_{l}\right|=\sqrt{2 P_{l} \kappa / \hbar \omega_{l}}$.

The dynamics of the tripartite atom-field-mirror system can be described by a set of nonlinear Langevin equations in which dissipation and fluctuation terms are added to the Heisenberg equations of motion derived from the Hamiltonian of Eqs. (11)-(2) 29]. However, we consider a simplified version of such equations, which is valid in the low atomic excitation limit, i.e., when all the atoms are initially prepared in their ground state, so that $S_{z} \simeq\left\langle S_{z}\right\rangle \simeq-N_{a}$ and this condition is not appreciably altered by the interaction with the cavity. This is satisfied when the excitation probability of a single atom is small. In this limit the dynamics of the atomic polarization can be described in terms of bosonic operators: in fact if one defines the atomic annihilation operator $c=S_{-} / \sqrt{\left|\left\langle S_{z}\right\rangle\right|}$, one can see that it satisfies the usual bosonic commutation relation $\left[c, c^{\dagger}\right]=1$ [30]. In the frame rotating at the laser frequency $\omega_{l}$ for the atomcavity system, the quantum Langevin equations can then be written as

$$
\begin{aligned}
& \dot{q}=\omega_{m} p \\
& \dot{p}=-\omega_{m} q-\gamma_{m} p+G_{0} a^{\dagger} a+\xi \\
& \dot{a}=-\left(\kappa+i \Delta_{f}\right) a+i G_{0} a q-i G_{a} c+E_{l}+\sqrt{2 \kappa} a_{i n}, \\
& \dot{c}=-\left(\gamma_{a}+i \Delta_{a}\right) c-i G_{a} a+\sqrt{2 \gamma_{a}} F_{c},
\end{aligned}
$$

where $\Delta_{f}=\omega_{c}-\omega_{l}$ and $\Delta_{a}=\omega_{a}-\omega_{l}$ are respectively the cavity and atomic detuning with respect to the laser, $G_{a}=g \sqrt{N_{a}}$, and $2 \gamma_{a}$ is the decay rate of the atomic excited level. The Langevin noise operators affecting the system have zero mean value, the Hermitian Brownian noise operator $\xi$ has correlation function $\left\langle\xi(t) \xi\left(t^{\prime}\right)\right\rangle=$ $\left(\gamma_{m} / 2 \pi \omega_{m}\right) \int d \omega e^{-i \omega\left(t-t^{\prime}\right)} \omega\left[\operatorname{coth}\left(\hbar \omega / 2 k_{B} T\right)+1\right]\left(k_{B}\right.$ is the Boltzmann constant and $T$ the temperature of the mechanical oscillator reservoir) 31], while the only nonvanishing correlation function of the noises affecting atoms and cavity field is $\left\langle a_{i n}(t) a_{i n}^{\dagger}\left(t^{\prime}\right)\right\rangle=$ $\left\langle F_{c}(t) F_{c}^{\dagger}\left(t^{\prime}\right)\right\rangle=\delta\left(t-t^{\prime}\right)$.

We now assume that the cavity is intensely driven, so that at the steady state, the intracavity field has a large amplitude $\alpha_{s}$, with $\left|\alpha_{s}\right| \gg 1$. However, the single-atom excitation probability is $g^{2}\left|\alpha_{s}\right|^{2} /\left(\Delta_{a}^{2}+\gamma_{a}^{2}\right)$ and since this probability has to be much smaller than one for the validity of the bosonic description of the atomic polarization, this imposes an upper bound to $\left|\alpha_{s}\right|$. Therefore the two conditions are simultaneously satisfied only if the atoms are weakly coupled to the cavity, $g^{2} \ll \Delta_{a}^{2}+\gamma_{a}^{2}$.

In the strong-driving limit, one has a semiclassical steady state; the corresponding mean values can be determined by setting the time derivatives to zero and factorizing the averages in Eqs. (3), and then solving the corresponding set of nonlinear algebraic equations. The resulting stationary values are $p_{s}=0, q_{s}=G_{0}\left|\alpha_{s}\right|^{2} / \omega_{m}$, $c_{s}=-i G_{a} \alpha_{s} /\left(\gamma_{a}+i \Delta_{a}\right)$, where the stationary intracavity field is the solution of the nonlinear equation $\alpha_{s}\left[\kappa+i \Delta_{f}-i G_{0}^{2}\left|\alpha_{s}\right|^{2} / \omega_{m}+G_{a}^{2} /\left(\gamma_{a}+i \Delta_{a}\right)\right]=E_{l}$. We are interested in establishing the presence of quantum correlations among atoms, field and mirror, at the steady state. This can be done by analyzing the dynamics of the quantum fluctuations of the system around the steady state. It is convenient to consider the vector of quadrature fluctuations $u=(\delta q, \delta p, \delta X, \delta Y, \delta x, \delta y)^{\top}$, where $\delta X \equiv\left(\delta a+\delta a^{\dagger}\right) / \sqrt{2}, \delta Y \equiv\left(\delta a-\delta a^{\dagger}\right) / i \sqrt{2}$, $\delta x \equiv\left(\delta c+\delta c^{\dagger}\right) / \sqrt{2}$, and $\delta y \equiv\left(\delta c-\delta c^{\dagger}\right) / i \sqrt{2}$, and linearize the quantum Langevin equations (3) around the steady state values. The resulting evolution equation for the fluctuation vector is

$$
\dot{u}=A u+n,
$$


where the drift matrix $A$ is given by

$$
A=\left(\begin{array}{cccccc}
0 & \omega_{m} & 0 & 0 & 0 & 0 \\
-\omega_{m} & -\gamma_{m} & G_{m} & 0 & 0 & 0 \\
0 & 0 & -\kappa & \Delta & 0 & G_{a} \\
G_{m} & 0 & -\Delta & -\kappa & -G_{a} & 0 \\
0 & 0 & 0 & G_{a} & -\gamma_{a} & \Delta_{a} \\
0 & 0 & -G_{a} & 0 & -\Delta_{a} & -\gamma_{a}
\end{array}\right)
$$

with the effective optomechanical coupling $G_{m}=$ $G_{0} \alpha_{s} \sqrt{2}$ (we have chosen the phase reference so that $\alpha_{s}$ can be taken real) and the effective cavity detuning $\Delta=\Delta_{f}-G_{m}^{2} / 2 \omega_{m}$. The vector of noises $n$ is given by $n=\left(0, \xi, \sqrt{2 \kappa} X_{i n}, \sqrt{2 \kappa} Y_{i n}, \sqrt{2 \gamma_{a}} x_{i n}, \sqrt{2 \gamma_{a}} y_{i n}\right)^{\top}$, where $X_{i n}=\left(a_{i n}+a_{i n}^{\dagger}\right) / \sqrt{2}, Y_{i n}=\left(a_{i n}-a_{i n}^{\dagger}\right) / i \sqrt{2}, x_{i n}=$ $\left(F_{c}+F_{c}^{\dagger}\right) / \sqrt{2}$ and $y_{i n}=\left(F_{c}-F_{c}^{\dagger}\right) / i \sqrt{2}$. Owing to the Gaussian nature of the quantum noise terms $\xi, a_{i n}$ and $F_{c}$, and to the linearization of the dynamics, the steady state of the quantum fluctuations of the system is a CV tripartite Gaussian state, which is completely determined by the $6 \times 6$ correlation matrix $(\mathrm{CM})$ $V_{i j}=\left\langle u_{i}(\infty) u_{j}(\infty)+u_{j}(\infty) u_{i}(\infty)\right\rangle / 2$. The Brownian noise $\xi(t)$ is not delta-correlated and therefore does not describe a Markovian process [31]. However, entanglement can be achieved only with a large mechanical quality factor, $\mathcal{Q}=\omega_{m} / \gamma_{m} \gg 1$. In this limit, $\xi(t)$ becomes delta-correlated [32], $\left\langle\xi(t) \xi\left(t^{\prime}\right)+\xi\left(t^{\prime}\right) \xi(t)\right\rangle / 2 \simeq$ $\gamma_{m}(2 \bar{n}+1) \delta\left(t-t^{\prime}\right)$, where $\bar{n}=\left(\exp \left\{\hbar \omega_{m} / k_{B} T\right\}-1\right)^{-1}$ is the mean vibrational number. In this Markovian limit, the steady state $\mathrm{CM}$ can be derived from the following equation [22, 33]

$$
A V+V A^{\top}=-D,
$$

where $D=\operatorname{Diag}\left[0, \gamma_{m}(2 \bar{n}+1), \kappa, \kappa, \gamma_{a}, \gamma_{a}\right]$ is the diffusion matrix stemming from the noise correlations.

We have solved Eq. (6) for the CM $V$ in a wide range of the parameters $G_{m}, G_{a}, \Delta$ and $\Delta_{a}$. We have studied first of all the stationary entanglement of the three possible bipartite subsystems, by quantifying it in terms of the logarithmic negativity [34] of bimodal Gaussian states. We will denote the logarithmic negativities for the mirror-atom, atom-field and mirror-field bimodal partitions with $E_{m a}, E_{a f}$ and $E_{m f}$, respectively.

The results on the behavior of the bipartite entanglement are shown in Fig. 2. We have considered experimentally feasible parameters [11, 13], i.e., an oscillator with $\omega_{m} / 2 \pi=10^{7} \mathrm{~Hz}, \mathcal{Q}=10^{5}$ and $m=10 \mathrm{ng}$ coupled to a cavity driven by a laser of power $P=35 \mathrm{~mW}$ at $\lambda_{l}=1064 \mathrm{~nm}$ (corresponding to $G_{m} / 2 \pi=8 \times 10^{6}$ $\mathrm{Hz}$ ), with length $L=1 \mathrm{~mm}$ and finesse $\mathcal{F}=3 \times 10^{4}$. The properties of the chosen working point of the cavity system are shown in Fig. 2a, showing the mirror-cavity mode logarithmic negativity and, in the inset, the effective mean excitation number of the mechanical oscillator, $n_{e f f}$, in the absence of the atoms, versus the normalized cavity detuning. The inset shows that we are
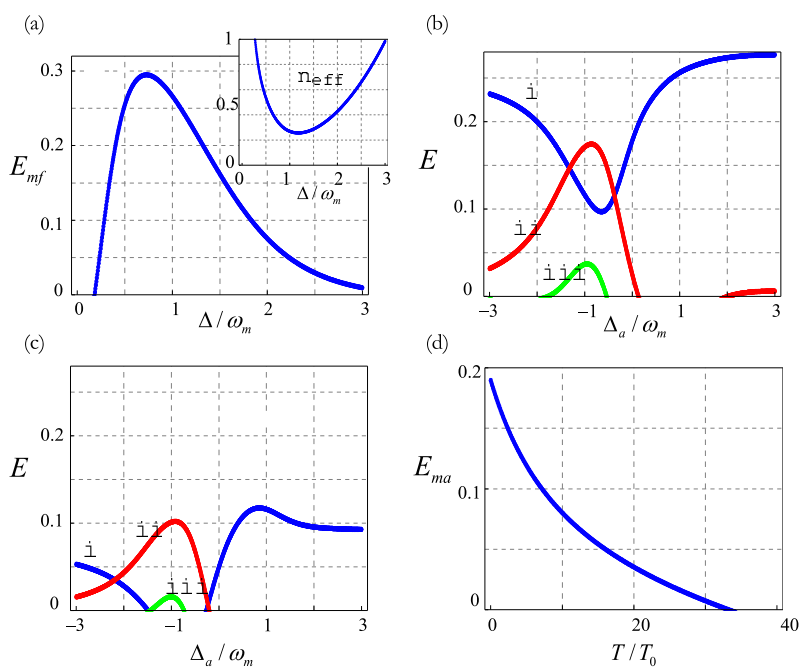

FIG. 2: (Color online) (a) Logarithmic negativity of the mirror-field subsystem versus the normalized cavity detuning in the absence of the atoms. Entanglement is maximized around the optimal cooling regime (shown in the inset) namely around $\Delta \simeq \omega_{m}$ (see text for the other parameter values). (b) Logarithmic negativity for the three bipartite entanglements as a function of the normalized atomic detuning (see text for the value of atomic parameters). At $\Delta_{a}=-\omega_{m}$ a decrease in the mirror-field ( $i$, blue line) entanglement is associated with an increase of the mirror-atoms ( $i i$, red line) and atoms-field (iii, green line) entanglement. (c) Same as in (b) but for the larger temperature $T=5 T_{0}$. (d) Temperature robustness of mirror-atoms entanglement up to $40 T_{0}(24 \mathrm{~K})$.

close to ground state cavity cooling of the mirror vibrational mode because $n_{\text {eff }}$ is decreased from the initial value $\bar{n}=1250$ (corresponding to a reservoir temperature $\left.T_{0}=0.6 \mathrm{~K}\right)$ to $n_{e f f} \simeq 0.2$ when $\Delta=\omega_{m}$, i.e., the cavity is resonant with the anti-Stokes sideband of the laser. This cooling regime allows to reach simultaneously a significant optomechanical entanglement. This can be understood in view of the results of [23, 35], where the entanglement between a vibrating mirror and the scattered optical sidebands is analyzed; when the mirror effective temperature is low enough one can have strong mirror-Stokes sideband entanglement. This latter entanglement is then exploited when the atomic ensemble is placed within the cavity. In Fig. 2(b)-(c), the logarithmic negativity of the three bipartite cases is plotted versus the normalized atomic detuning when $\gamma_{a} / 2 \pi=5 \times 10^{6}$ $\mathrm{Hz}$, and $G_{a} / 2 \pi=6 \times 10^{6} \mathrm{~Hz}$. It is evident that one has a sort of entanglement sharing: due to the presence of the atoms, the initial cavity-mirror entanglement is partially redistributed to the atom-mirror and atom-cavity subsystems and this effect is predominant when the atoms are resonant with the Stokes sideband $\left(\Delta_{a}=-\omega_{m}\right)$. It is remarkable that, in the chosen parameter regime, the largest stationary entanglement is the one between atoms and mirror which are only indirectly coupled. More- 
over, the nonzero atom-cavity entanglement appears only thanks to the effect of the mirror dynamics because in the bosonic approximation we are considering and with a fixed mirror, there would be no direct atom-cavity entanglement. We also notice that atom-mirror entanglement is instead not present at $\Delta_{a}=\omega_{m}$. This is due to the fact that the cavity-mirror entanglement is mostly carried by the Stokes sideband and that, when $\Delta_{a}=\omega_{m}$, mirror cavity-cooling is disturbed by the Antistokes photons being recycled in the cavity by the absorbing atoms.

Fig. 2(c) shows the same plot but at a higher temperature, $T=5 T_{0}=3 \mathrm{~K}$, showing that the three bipartite entanglements are quite robust with respect to thermal noise. This is studied in more detail in Fig. 2(d), where the atom-mirror entanglement at $\Delta_{a}=-\omega_{m}$ is plotted versus the reservoir temperature: such an entanglement vanishes only around $20 \mathrm{~K}$.

The simultaneous presence of all the three possible instances of bipartite entanglement witnesses the strong correlation between the atoms, the intracavity field, and the mechanical resonator at the steady state. This is also confirmed by the fact that such a state is a fully inseparable tripartite CV entangled state in the parameter regime of Fig. 2, for a wide range of atomic detuning $\left(-3 \omega_{m}<\Delta_{a}<3 \omega_{m}\right)$ and up to temperatures of about $30 \mathrm{~K}$. This has been checked by applying the results of Ref. 36], which provide a necessary and sufficient criterion for the determination of the entanglement class of a tripartite CV Gaussian state.

We notice that the chosen parameters correspond to a small cavity mode volume $\left(V \simeq 10^{-12} \mathrm{~m}^{3}\right)$, implying that for a dipole transition, $g$ is not small. Therefore the assumed weak coupling condition $g^{2} \ll \Delta_{a}^{2}+\gamma_{a}^{2}$ can be satisfied only if $g$ represents a much smaller, time averaged, coupling constant. This holds for example for an atomic vapor cell much larger than the cavity mode: if the (hot) atoms move in a cylindrical cell with axis orthogonal to the cavity axis, with diameter $\sim 0.5 \mathrm{~mm}$ and height $\sim 1 \mathrm{~cm}$, they will roughly spend only one thousandth of their time within the cavity mode region. This yields an effective $g \sim 10^{4} \mathrm{~Hz}$, so that the assumptions made here hold, and the chosen value $G_{a} / 2 \pi=6 \times 10^{6}$ $\mathrm{Hz}$ can be obtained with $N_{a} \sim 10^{7}$. An alternative solution could be choosing a cold atomic ensemble and a dipole-forbidden transition.

The entanglement properties of the steady state of the tripartite system can be verified by experimentally measuring the corresponding CM. This can be done by combining existing experimental techniques. The cavity field quadratures can be measured directly by homodyning the cavity output, while the mechanical position and momentum can be measured with the setup proposed in [22], in which by adjusting the detuning and bandwidth of an additional adjacent cavity, both position and momentum of the mirror can be measured by homodyning the output of this second cavity. Finally, the atomic polarization quadratures $x$ and $y$ (proportional to $S_{x}$ and $S_{y}$ ) can be measured by adopting the same scheme of Ref. [37], i.e., by making a Stokes parameter measurement of a laser beam, shined transversal to the cavity and to the cell and off-resonantly tuned to another atomic transition.

In conclusion we have proposed a scheme for the realization of a hybrid quantum correlated tripartite system formed by a cavity mode, an atomic ensemble inside it, and a vibrational mode of one cavity mirror. We have shown that, in an experimentally accessible parameter regime, the steady state of the system shows both tripartite and bipartite CV entanglement. The realization of such a scheme will open new perspectives for the realization of quantum interfaces and memories for $\mathrm{CV}$ quantum information processing and also for quantum-limited displacement measurements.

This work was supported by the European Commission (programs QAP and SCALA), and by the Italian Ministry for University and Research (PRIN-2005 2005024254).

[1] I. Bloch, Nat. Phys. 1, 23 (2005).

[2] D. Porras, and J.I. Cirac, Phys. Rev. Lett. 92, 207901 (2004).

[3] G. Ciaramicoli, I. Marzoli, and P. Tombesi, Phys. Rev. A 75, 032348 (2007).

[4] A. Wallraff et al., Nature 431, 162 (2004).

[5] L. Tian and P. Zoller, Phys. Rev. Lett. 93, 266403 (2004).

[6] L. Tian et al., Phys. Rev. Lett. 92, 247902 (2004).

[7] P. Treutlein et al., Phys. Rev. Lett. 99, 140403 (2007).

[8] P. F. Cohadon, A. Heidmann, and M. Pinard, Phys. Rev. Lett. 83, 3174 (1999).

[9] C. H. Metzger and K. Karrai, Nature (London), 432, 1002 (2004).

[10] T. J. Kippenberg et al. Phys. Rev. Lett. 95033901 (2005).

[11] S. Gigan et al. Nature (London) 444, 67 (2006).

[12] O. Arcizet et al., Phys. Rev. Lett. 97, 133601 (2006).

[13] O. Arcizet et al. Nature (London) 444, 71 (2006).

[14] D. Kleckner and D. Bouwmeester, Nature (London) 444, 75 (2006)

[15] A. Schliesser et al., Phys. Rev. Lett. 97243905 (2006); A. Schliesser et al., arXiv:0709.4036v1[quant-ph].

[16] T. Corbitt et al., Phys. Rev. Lett. 99, 160801 (2007).

[17] M. Poggio et al., Phys. Rev. Lett. 99, 017201 (2007).

[18] J. G. E. Harris et al., Rev. Sci. Instrum. 78, 013107 (2007); J. D. Thompson et al., arXiv:0707.1724v2[quant$\mathrm{ph}]$.

[19] S. Mancini et al., Phys. Rev. Lett. 88, 120401 (2002).

[20] M. Pinard et al., Europhys. Lett. 72, 747 (2005).

[21] S. Pirandola et al., Phys. Rev. Lett. 97, 150403 (2006).

[22] D. Vitali et al., Phys. Rev. Lett. 98, 030405 (2007).

[23] S. Mancini, D. Vitali, and P. Tombesi, Phys. Rev. Lett. 90, 137901 (2003).

[24] I. Wilson-Rae et al., Phys. Rev. Lett. 99, 093901 (2007).

[25] F. Marquardt et al., Phys. Rev. Lett. 99, 093902 (2007).

[26] C. Genes et al., arXiv:0705.1728v3 [quant-ph].

[27] A. Dantan et al., arXiv:0707.2038v1 [quant-ph]. 
[28] M. Tavis, F. W. Cummings, Phys. Rev. 170, 379 (1968).

[29] C. W. Gardiner and P. Zoller, Quantum Noise, (Springer, Berlin, 2000).

[30] T. Holstein, and H. Primakoff, Phys. Rev. 58, 1098 (1940).

[31] V. Giovannetti, D. Vitali, Phys. Rev. A 63, 023812 (2001).

[32] R. Benguria, and M. Kac, Phys. Rev. Lett, 46, 1 (1981).

[33] P. C. Parks and V. Hahn, Stability Theory, Prentice Hall,
New York, 1993

[34] J. Eisert, PhD thesis (Potsdam, 2001); G. Vidal and R.F. Werner, Phys. Rev. A 65, 032314 (2002); G. Adesso et al., Phys. Rev. A 70, 022318 (2004).

[35] S. Pirandola et al., Phys. Rev. A 68, 062317 (2003).

[36] G. Giedke et al., A 64, 052303 (2001).

[37] J. Sherson et al., Nature 443, 557 (2006). 